\title{
Ink-jetted Silver/Copper conductors for printed RFID applications
}

Steven K. Volkman, Yunan Pei, David Redinger, Shong Yin, and Vivek Subramanian. Department of Electrical Engineering, University of California Berkeley, Berkeley, CA 94720-1770, U.S.A.

\begin{abstract}
Low-resistance printed conductors are crucial for the development of ultra-low cost electronic systems such as radio frequency identification tags. Low resistance conductors are required to enable the fabrication of high-Q inductors, capacitors, tuned circuits, and interconnects. Furthermore, conductors of appropriate workfunction are also required to enable fabrication of printed Schottky diodes, necessary for rectification in RFID circuits. Last year, we demonstrated the formation of low-resistance conductive printed structures using gold nanoparticles. Here we demonstrate, for the first time, technologies for formation of printed conductors using silver and copper nanoparticles. These are particularly advantageous for several reasons. First, both silver and copper offer a $2 \mathrm{X}$ reduction in sheet resistance over gold, resulting in improved interconnect performance and inductor Q. Second, the material costs associated with both silver and copper are expected to be significantly cheaper than gold. Third, the workfunction of silver enables the fabrication of all-printed Schottky diodes with a silver rectifying contact to many common printable organic semiconductors.

Solutions of organic-encapsulated silver and copper nanoparticles may be printed and subsequently annealed to form low-resistance conductor patterns. We describe novel processes for forming silver and copper nanoparticles, and discuss the optimization of the printing/annealing processes to demonstrate plastic-compatible low-resistance conductors. By optimizing both the size of the nanoparticle and the encapsulant sublimation kinetics, it is possible to produce particles that anneal at low-temperatures $\left(<150{ }^{\circ} \mathrm{C}\right)$ to form continuous films having low resistivity and appropriate workfunction for formation of rectifying contacts. This represents a major component required for allprinted RFID.
\end{abstract}

\section{INDRODUCTION}

In recent years, there has been tremendous interest in flexible electronics. Besides the obvious applications of flexible electronics in flat panel displays [1], flexible circuits are also promising for use in such applications as radio frequency identification (RFID) tags [2], low cost sensors [3], and other disposable electronic devices. In particular, devices based on organic semiconductors are considered to be very promising for these applications since they may potentially be fabricated entirely using printing technologies [4], eliminating the need for such major cost points as lithography, vacuum processing including physical vapor deposition, plasma etching, and chemical vapor deposition, while simultaneously allowing the use of reel-to-reel processing, resulting in reduced substrate handling and clean room costs as well. Furthermore, since printing is inherently additive in nature, material and disposal costs are also expected to be reduced, resulting in an extremely low net system cost. 
Advances in organic semiconductor technology has been astounding, with demonstrations of fabricated RFIDs operating above $1 \mathrm{MHz}$ [5]. However, the ability to replicate such promising results using low-cost reel-to-reel methods is still in its infancy. With the improvements in printable semiconductor technology [6-7], printable conductor technology must keep pace. RFID applications will require the printing of high-Q inductors, transformers, and tuned circuits [8]. We have previously reported such a technology using gold nano-particles as a soluble gold precursor. Gold has an adequate work-function match to many organic semi-conductors including pentacene. However, its workfunction is not appropriate for the formation of diodes, which are necessary for rectification in RFID circuits. Silver and copper nano-particles are less expensive than gold; their films have the potential for increased conductivity over gold, and the 0.7-0.8 $\mathrm{eV}$ lower workfunction of silver and copper make them ideal for printed organic diodes. It is this potential for a printed organic diode that makes a soluble precursor to silver and copper films of paramount importance.

Similar to our development of a printable gold conductor, we harness the unique characteristics of nano-particles to form solution deposited silver and copper films at low temperatures. It has been known for some time that metal nano-particles have reduced melting and sintering temperatures when compared to their bulk counterparts [9]. Monolayer protected nano-particles of silver and copper can be readily dissolved into non-polar solvents such as toluene or even polar-protic solvents such as 2-propanol. They can then be deposited on a variety of substrates, including plastics and glass, and then heated to form conductive films. We report here our initial inquiries into printable silver and copper films and develop optimization strategies to further enhance performance.

\section{EXPERIMENTAL DETAILS}

The experiment process consists of three stages: a novel synthesis of both silver and copper nano-particles, the analysis of the anneal process, and the printing of conductive lines.

\section{$\underline{\text { Nano-particle synthesis }}$}

Silver nano particles were formed by adding $\mathrm{NaBH}_{4}(0.5 \mathrm{~g})$ dissolved in 2propanol $(85 \mathrm{~mL})$ to a solution of $\mathrm{AgNO}_{3}(0.5 \mathrm{~g})$ and alkane thiol in 2-propanol $(85 \mathrm{~mL})$. The size of the nano-particles was controlled by the ratio of alkanethiol to $\mathrm{AgNO}_{3}$

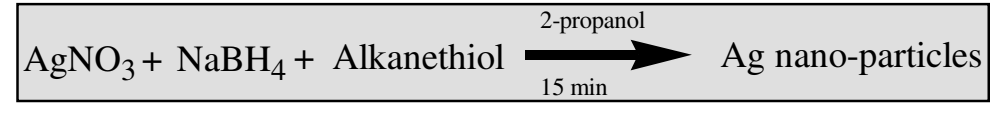

Figure 1: Synthesis of silver nano-particles.

Copper nano-particles were formed by adding $\mathrm{NaBH}_{4}(0.22 \mathrm{~g})$ dissolved in 2propanol $(85 \mathrm{~mL})$ to a solution of $\mathrm{Cu}\left(\mathrm{NO}_{3}\right)_{2}-\mathrm{H}_{2} \mathrm{O}(0.1 \mathrm{~g})$ and hexylamine in 2-propanol $(85 \mathrm{~mL})$ over a period of two hours. 


$\mathrm{CuCl}_{2}+\mathrm{NaBH}_{4}+$ Alkylamine $\underset{2 \text { hrs }}{\stackrel{2-\text { propanol }}{\longrightarrow}} \mathrm{Cu}$ nano-particles

Figure 2: Synthesis of copper nano-particles.

Once the reactions have concluded, the solutions are purified by filtering to remove all un-reacted starting materials.

\section{$\underline{\text { Anneal Process }}$}

As discussed above, a thermal anneal process is used to convert the nano-particles to a metallic film. We synthesized nano-particles encapsulated by various organic molecules to determine the encapsulants' effect on anneal temperatures, which is obviously extremely important when printing on substrates with a low thermal budget. Specifically: silver nano-particles were formed using butane thiol, hexane thiol, and dodecane thiol. Copper nano-particles were formed using hexylamine. The silver nanoparticles were annealed in air, and the copper nano-particles were annealed in a 20:80 $\mathrm{H}_{2} / \mathrm{N}_{2}$ environment.

\section{$\underline{\text { Ink-jetted Films }}$}

Using the conditions developed above, films were formed with inkjetted nanoparticles. All inkjet experiments were performed using a custom inkjet system, shown in Figure 3. The overall testbed consists of translation stages, inkjet dispensers, a hot chuck for heating and cooling the substrate, and software to control the various systems. For all experiments, we used piezoelectric heads manufactured by Microfab, Inc., with nozzle diameters varying from $30 \mu \mathrm{m}$ to $60 \mu \mathrm{m}$. Custom software was used to provide translation and head control.

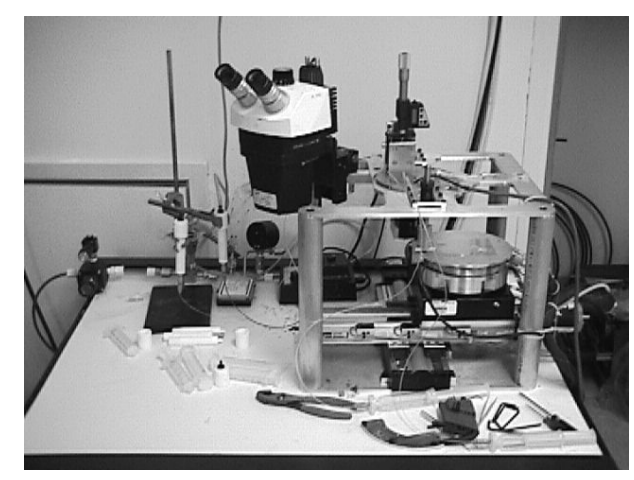

Figure 3: The custom inkjet system for printed electronics fabrication.

The nano-particles were dissolved in butylbenzene and ink-jetted onto plastic substrates. The resulting sheet resistance was extracted. 


\section{RESULTS}

For nano-particles deposited onto a substrate to become conductive, two events must occur. First, the organic encapsulant that surrounds the nano-particle must be removed in a burn-off event. Second, the nano-particles must be raised to a temperature that allows for them to sinter or melt in a continuous film; this is referred to as the conduction temperature. If the first event does not occur, insulating alkane chains separate the nano-particles, in which only small tunneling currents are possible. If the second event does not occur then the film remains a mono-dispersed collection of individual nano-particles that have little interaction.

For silver, the temperature at which the deposited nano-particles were annealed into a conductive film was independent of thiol chain length. This is dissimilar to what we had previously observed with gold in which the conduction temperature could be reduced with the use of shorter alkane thiols. Furthermore, we find that the temperature at which the thiol encapsulant is removed corresponds exactly to the conduction temperature. This is also unlike the results we had previously observed for gold in which the thiol burn off temperature could be as much as $50^{\circ} \mathrm{C}$ lower than the conduction temperature [10].

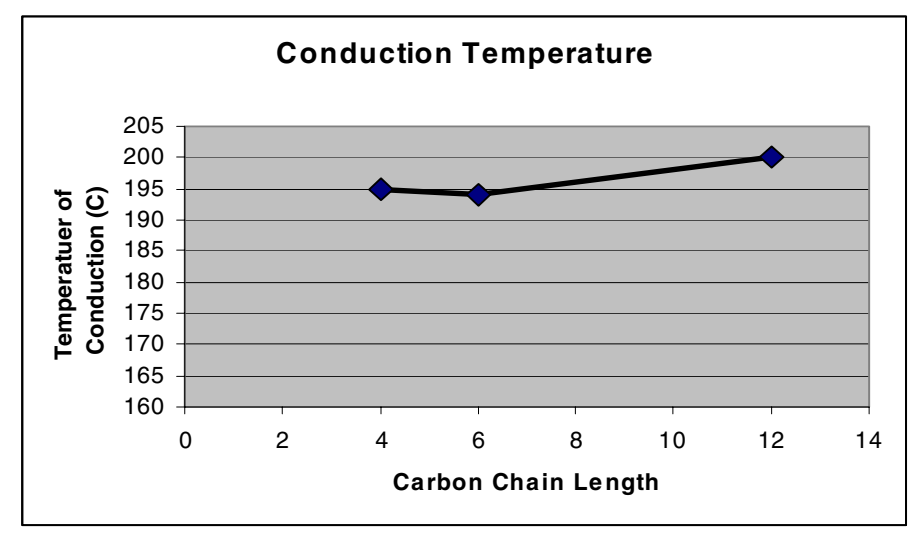

Figure 4: The effect of thiol chain length on conduction temperature.

For copper, conductive films could be formed at temperatures $<150{ }^{\circ} \mathrm{C}$. The copper nano-particles themselves seemed to be well behaved as seen in Figure 5.
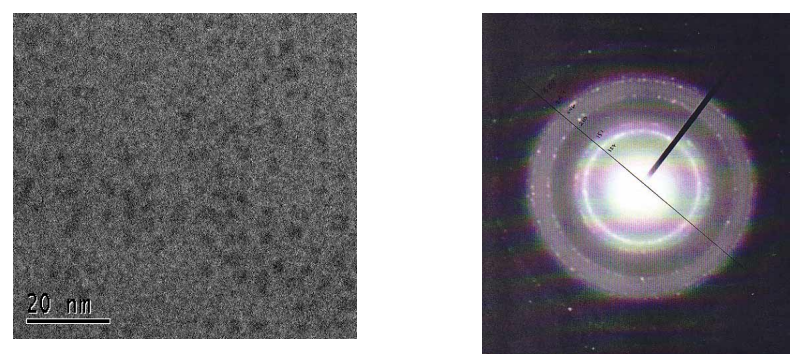

Figure 5: Transmission electron micrograph (left) and electron diffraction pattern (right) for copper nano-particles. 
Both silver and copper nano-particles were ink-jet printed to form films on plastics. The sheet resistance under un-optimized conditions is $100 \mathrm{~m} \Omega / \mathrm{square}$ for films a few thousands of angstroms in thickness. Surface roughness prevented accurate film thickness measurements, which will be resolved with optimization of the printing conditions.
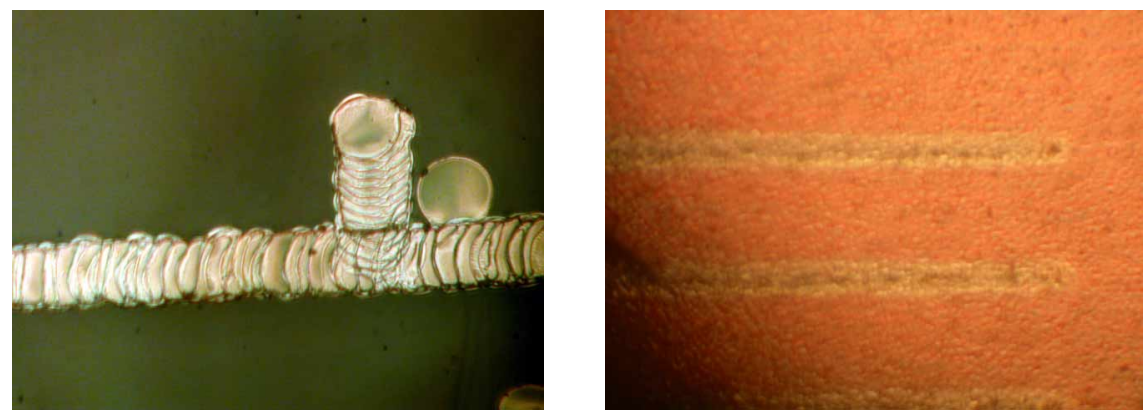

Figure 6: Ink-jet printed silver (left ) and copper (right) films

\section{DISCUSSION}

For thiol encapsulated Ag nano-particles we see very little dependence of the temperature on which a conductive film is formed and the length of the alkanethiol chain. This is unlike what we previously reported for Au. Also, unlike the Au, there is no difference in the temperature at which burn off and conduction occurs. This suggests both that the dominant factor determining burn-off is not the length of the thiol chain but the thiol functional group itself and that burn-off, not the sintering of the nano-particles, is the limiting factor for the conduction temperature.

For both $\mathrm{Cu}$ and $\mathrm{Ag}$ nano-particles we observed conductive films upon inkjetting. Further optimization of print conditions is necessary for reduction in film resistivity.

\section{CONCLUSION}

$\mathrm{Ag}$ and $\mathrm{Cu}$ nano-particles were successfully processed into conductive films. Such processes are compatible with ink-jet printing and can easily be incorporated into the solution based manufacturing of electronic circuits. The dependency of the sinter temperature on the encapsulant burn-off event for $\mathrm{Ag}$, as well as the strong influence of the functional group, suggests that alternant functional groups should be examined as encapsulants to lower the conduction temperature. Our experience with Au indicates that film roughness and cracking issues can be resolved through optimization of deposition conditions; a similar analysis of the $\mathrm{Ag}$ and $\mathrm{Cu}$ system is necessary to reduce resistances in the deposited films.

\section{ACKNOWLEDGEMENTS}

The authors would like to thank the National Center of Electron Microscopy at the Lawrence Berkeley National Laboratory for the use of their Transmission Electron 
Microscopes. Funding for this work was supported by grants from the Environmental Protection Agency and the National Science Foundation.

\section{REFERENCES}

[1] H. Klauk, B. D'Andrade, and T. N. Jackson, in the 57th Annual Device Research Conference Digest, IEEE, p. 162, (1999).

[2] B. K. Crone, A. Dodabalapur, R. Sarpeshkar, R. W. Filas, Y.-Y. Lin, and Z. Bao, Appl. Phys. Lett., 89, 5125 (2001).

[3] B. Crone, A. Dodabalapur, A. Gelperin, L. Torsi, H. E. Katz, and A. J. Lovinger, Appl. Phys. Lett., 78, 2229 (2001).

[4] V. Subramanian, Paper presented at the Half Moon Bay Maskless Lithography

Workshop, DARPA/SRC, Half Moon Bay, CA, Nov 9-10, 2000.

[5] P.F. Baude et al., Tech. Dig. IEDM 2003, p.191.

[6] Afzali et al, JACS Comm., 2002.

[7] S. Volkman, S. Molesa, B. Mattis, P. C. Chang, and V. Subramanian, MRS Proceedings Volume 769, H11.7, 2003.

[8] K. Finkenzeller, RFID Handbook, John Wiley \& Sons, New York (1999).

[9] D. C. Huang, F. Liao, and V. Subramanian, in Proceedings of the Materials Research

Society Spring 2002 Meeting, MRS, (2002).

[10] D. Huang, F. Liao, S. Molesa, D. Redinger, and V. Subramanian, Journal of the electrochemical society, 150, pp. 412-417, 2003. 